\title{
Оптимизация вертикально-излучающего лазера с внутрирезонаторными металлическими слоями
}

\author{
(C) А.А. Лазаренко ${ }^{1,2}$, К.А. Иванов ${ }^{1-3, \uparrow}$, А.Р. Губайдуллин ${ }^{1,2}$, М.А. Калитеевский ${ }^{1,2,4}$ \\ ${ }^{1}$ Санкт-Петербургский Академический университет Российской академии наук, \\ 194021 Санкт-Петербург, Россия \\ ${ }^{2}$ Университет ИТМО, \\ 197101 Санкт-Петербург, Россия \\ ${ }^{3}$ Санкт-Петербургский научный центр Российской академии наук, \\ 199034 Санкт-Петербург, Россия \\ ${ }^{4}$ Физико-технический институт им. А.Ф. Иоффе Российской академии наук, \\ 194021 Санкт-Петербург, Россия \\ ฯ E-mail: kivanov@corp.ifmo.ru
}

(Получена 28 сентября 2016 г. Принята к печати 7 октября 2016 г.)

Исследована зависимость затухания собственной оптической моды вертикально-излучающего лазера с внутрирезонаторными металлическими контактами от геометрических параметров системы. Показано, что наименьшее затухание имеет место для частоты собственной моды резонатора, отличной от брэгговской частоты зеркал. Методом $S$-квантования рассчитана вероятность спонтанной эмиссии, и показано, что для высокоэнергетической нечетной моды вероятность спонтанной эмиссии увеличивается на 2 порядка по сравнению с вероятностью эмиссии в свободном пространстве.

DOI: $10.21883 /$ FTP.2017.04.44350.8419

Локализованные состояния электромагнитного поля на границе металла и брэгговского отражателя - таммовские плазмоны (ТП) с момента их теоретического предсказания $[1,2]$ и экспериментального обнаружения $[3,4]$ являются объектом интенсивных исследований. Применения ТП связаны с возможностью их применения для локализации света в латеральном направлении в вертикальных микрорезонаторах [5] и использования структур на их основе для создания лазеров [6,7], источников одиночных фотонов [8] и других приборов. Слои металла (как правило, серебра или золота), используемые в структурах с ТП, поглощают свет, что ограничивает применимость металлических элементов в оптоэлектронных приборах. Проблема поглощения света в металлических элементах лазеров может быть решена путем усиления взаимодействия света с активной средой в „субволновых“ лазерах на основе металлических нанорезонаторов [9], при этом до недавнего времени было принято считать, что использование металлических слоев в качестве зеркал для полупроводниковых лазеров на основе вертикальных или планарных резонаторов Фабри-Перо должно приводить к деградации структур.

Недавно было показано, что особенности пространственного распределения поля в структурах с ТП позволяют решить проблему поглощения света в металле. В частности, в структурах с активной областью из органического материала, соединенной со слоем серебpa, наблюдалась макроскопическая оптическая когерентность [10]. Кроме того, в микрорезонаторах с внутрирезонаторными металлическими слоями [11] было экспе-
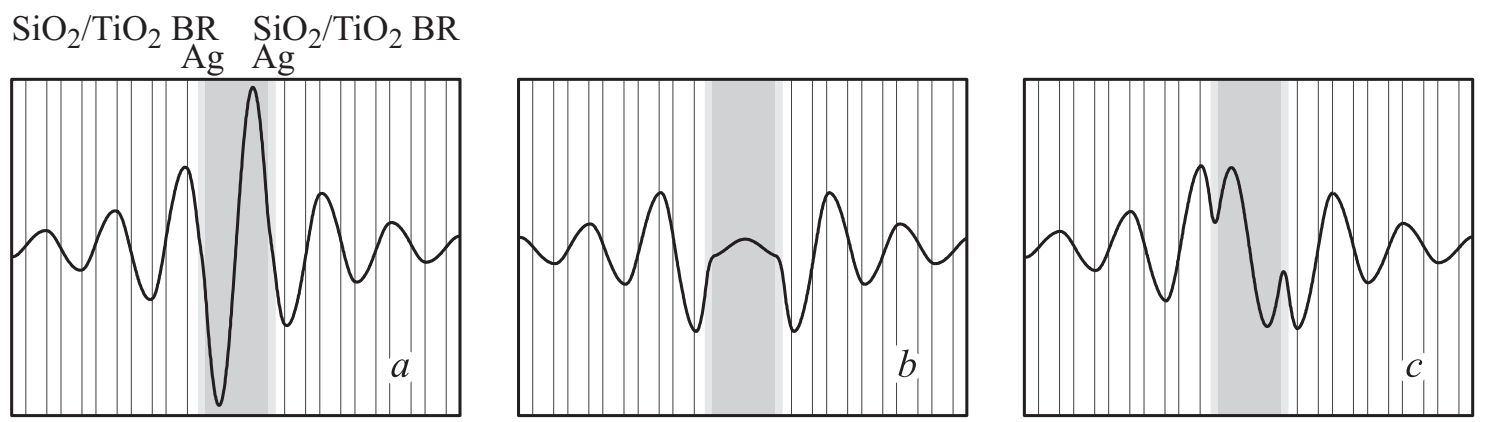

Active area

Рис. 1. Схема структуры: два слоя серебра толщиной 40 нм расположены на границах брэгговских зеркал микрорезонаторов и центральной активной области. Толщина слоев $\mathrm{TiO}_{2}$ и $\mathrm{SiO}_{2}$ в брэгговских зеркалах выбрана так, чтобы брэгговская длина волны равнялась 630 нм. Сплошной линией показаны профили электрического поля для собственных мод с комплексными частотами: $a-(2043-i 3.252)$ мэВ (мода с минимальным затуханием и максимальным перекрытием с активной областью); $b-(1969-i 8.110)$ мэВ; $c-(1929-i 10.66)$ мэВ. 


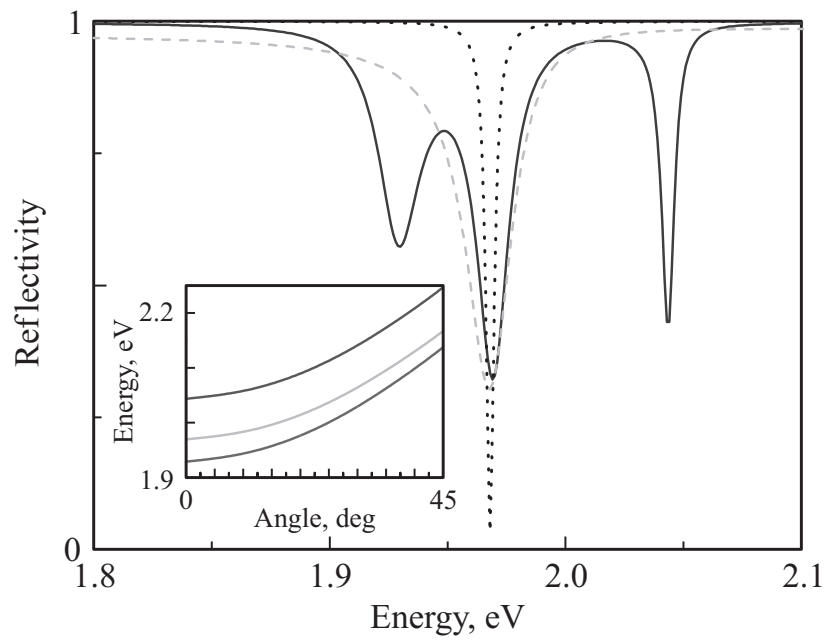

Pис. 2. Спектры отражения структуры, показанной на рис. 1 (сплошная линия). Для сравнения, пунктирной и штриховой линиями показаны спектры отражения от микрорезонатора без металлических слоев (пунктирная линия) и одиночного брэгговского отражателя с металлическим слоем (одиночный таммовский плазмон, пунктирная линия) соответственно. На вставке показаны зависимости частот трех гибридных мод от угла падения.

риментально продемонстрировано существенное снижение поглощения в металлических слоях. Такое снижение поглощения достигается дизайном профиля собственной оптической моды микрорезонатра с ТП, при котором тонкому слою металла соответствует узел электрического поля собственной моды. Данная работа посвящена исследованию возможности уменьшения затухания оптических мод, сконструированных с использованием ТП в микрорезонаторе с металлическими контактами, и исследованию скорости спонтанной эмиссии в таких структурах.
Схема структуры показана на рис. 1. Активная область, состоящая из органического материала DCM + Alq3 [12], заключена между двумя слоями серебра и брэгговскими отражателями из четвертьволновых слоев $\mathrm{SiO}_{2} / \mathrm{TiO}_{2}$. На границах металла и брэгговского отражателя локализованы ТП. Кроме того, мода Фабри-Перо локализована между двумя слоями серебра. Если частоты всех трех мод (двух ТП и моды Фабри-Перо) близки, то моды гибридизуются и расщепляются, при этом величина расщепления определяется толщиной внутрирезонаторных слоев металла. В связи с конечной глубиной проникновения поля в металл, фаза коэффициента отражения света от металла отлична от $\pi$ и зависит от частоты. Поэтому, для того чтобы частота таммовского плазмона соответствовала центру запрещенной зоны брэгговского отражателя, необходимо, чтобы ширина слоя брэгговского отражателя, прилегающего к металлу, была отлична от четвертьволновой.

На рис. 2 показаны спектры отражения от структуры, параметры которой подобраны таким образом, чтобы частоты моды Фабри-Перо, локализованной между

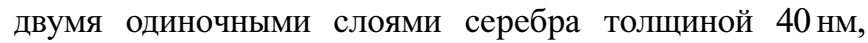
частоты одиночных ТП соответствовали брэгговской частоте. Три провала в спектре соответствуют трем гибридным модам, и можно видеть, что ширины провалов сильно различаются, что указывает на различную величину затухания мод. Для сравнения показаны спектры отражения, соответствующие микрорезонатору без металлических слоев и одиночному таммовскому плазмону. Ширина каждой из гибридных мод меньше, чем ширина одиночного ТП, но больше, чем ширина моды микрорезонатора без металлических слоев. Частоты всех гибридных мод демонстрируют параболическую зависимость от угла падения, как показано на вставке к рис. 2. Три гибридные моды имеют разный пространственный профиль, и электрическое поле моды, представляющей интерес для создания лазера (показана
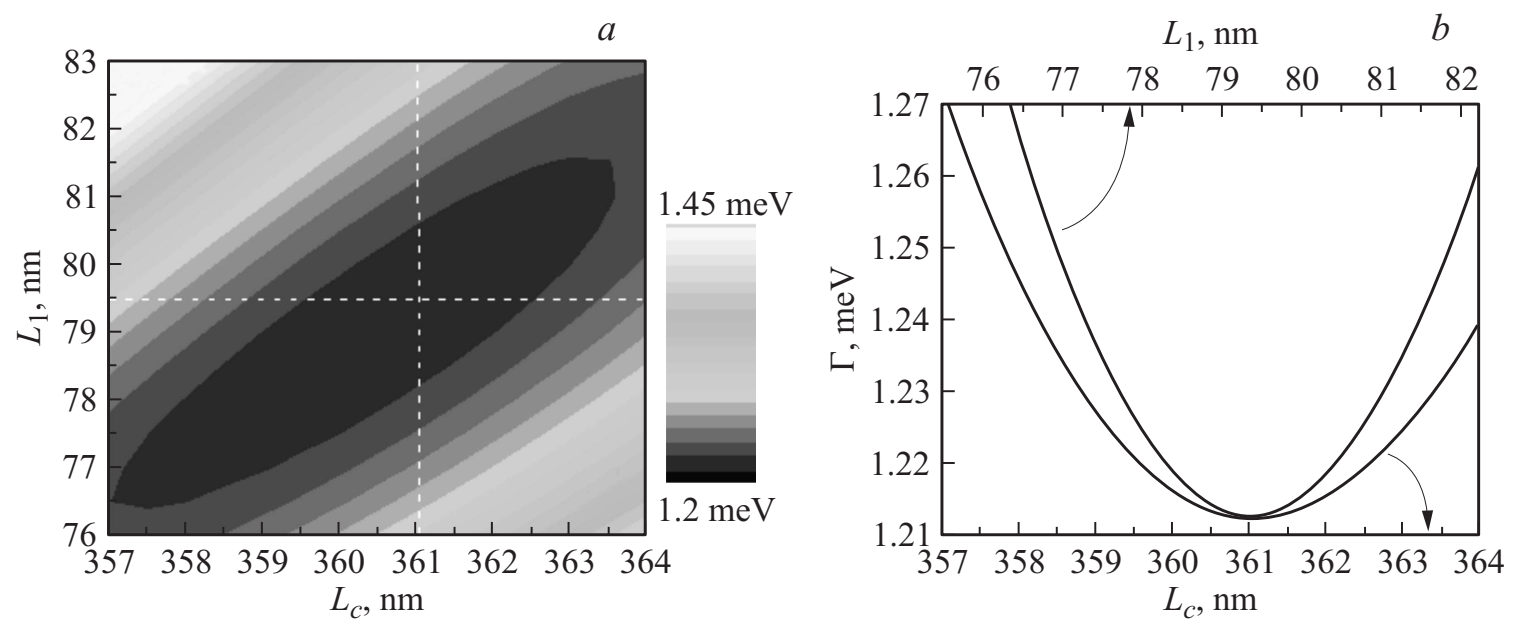

Рис. 3. $a$ - зависимость затухания гибридной моды, показанной на рис. $1, a$, от толщины активной области $L_{c}$ и фазосогласующих слоев $L_{1} . b$ - зависимость затухания моды от $L_{c}$ при фиксированном $L_{1}$, нижняя шкала (от $L_{1}$ при фиксированном $L_{c}$, верхняя шкала). Фиксированные значения $L_{1}$ и $L_{c}$ отмечены штриховыми линиями на рис. $3, a$. 


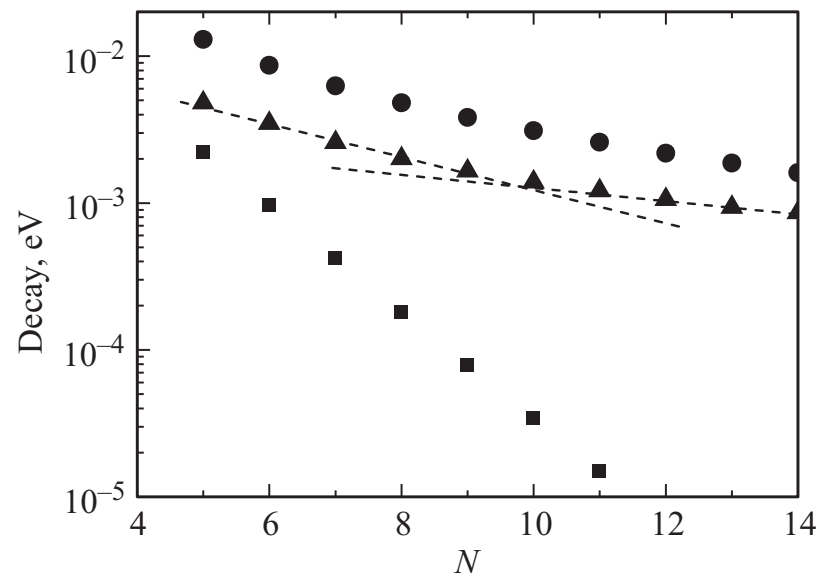

Рис. 4. Зависимость затухания оптической моды, показанной на рис. 1, в микрорезонаторе без металлических слоев (квадраты), в микрорезонаторе с металлическими слоями, показанном на рис. 3 (треугольники), и одиночного таммовского плазмона (круги). $N$ - число пар слоев в брэгговском отражателе.

на рис. $3, a)$, должно обладать наименьшим перекрытием с металлическими слоями и максимальным перекрытием с активной областью. Минимизации затухания моды типа А можно достичь, варьируя толщину активной области $L_{c}$ и толщины фазосогласующих слоев $L_{1}$.

Затухание собственной моды может быть рассчитано путем решения уравнений Максвелла методом матриц переноса с постановкой граничных условий, соответствующих убегающим волнам [13]. Оптимальная мода должна обладать минимальным значением затухания, определяемым мнимой частью собственной частоты, рассчитанной методом убегающих волн.

На рис. 3, $a$ показана зависимость затухания гибридной моды, локализованной в активной области микрорезонатора, от толщины полости микрорезонаторов и фазосогласующих слоев. Можно видеть, что для значений толщин $L_{c}=361$ нм и $L_{1}=79.5$ величина затухания составляет 1.2 мЭв. Отметим, что минимальное затухание достигается в случае, когда толщина полости микрорезонатора отлична от целого числа полуволн. Профиль моды с минимальным затуханием показан на рис. $1, a$, ее энергия составляет $(2043-i 3.252)$ мэВ. Электрическое поле этой моды обладает минимальным перекрытием с металлическими слоями структуры. На рис. $1, b$ и 1, $c$ показаны профили электрического поля для мод с комплексными собственными энергиями $(1969-i 8.110)$ и $(1929-i 10.66)$ мэВ. Можно видеть, что для данных мод перекрытие с активной областью меньше, а с металлическими слоями - больше, чем для моды, изображенной на рис. 1, $a$. Таким образом, структура предоставляет возможность для селекции мод: порог генерации для моды, изображенной на рис. 1, $a$, достигается значительно раньше, чем для остальных гибридных мод.

В обычных микрорезонаторах, без металлических слоев, затухание собственной моды экспоненциально падает при увеличении толщины зеркал, как показано на рис. 4. Для одиночного ТП увеличение ширины зеркала после некоторого предела не приводит к повышению добротности структуры вследствие поглощения в металле. В случае структуры, для моды, изображенной на рис. $1, a$, затухание существенно меньше, чем для ТП, но при этом существует оптимальная толщина зеркал, при превышении которой дальнейшее увеличение толщины зеркала не приводит к существенному уменьшению затухания, как показано на рис. 4.

На рис. 5, $a, b$ показана вероятность спонтанной эмиссии света для диполя, помещенного в пучность электрического поля моды, изображенной на рис. 1, $a$, рассчитанная методом $S$-квантования [14]. Для сравнения на рис. 5, $c$ приведена аналогичная величина для диполя в центре структуры, что соответствует пучности поля моды на рис. $1, b$. Можно видеть, что на рис. 5, $a, b$ проявляются только две моды (изображенные на рис. 1, $a$ и $c$, нечетные моды). В то же время для диполя, помещенного в центр структуры, проявляется мода, изображенная на рис. $1, b$ (четная мода). Можно видеть, что степень усиления спонтанной эмиссии на частоте
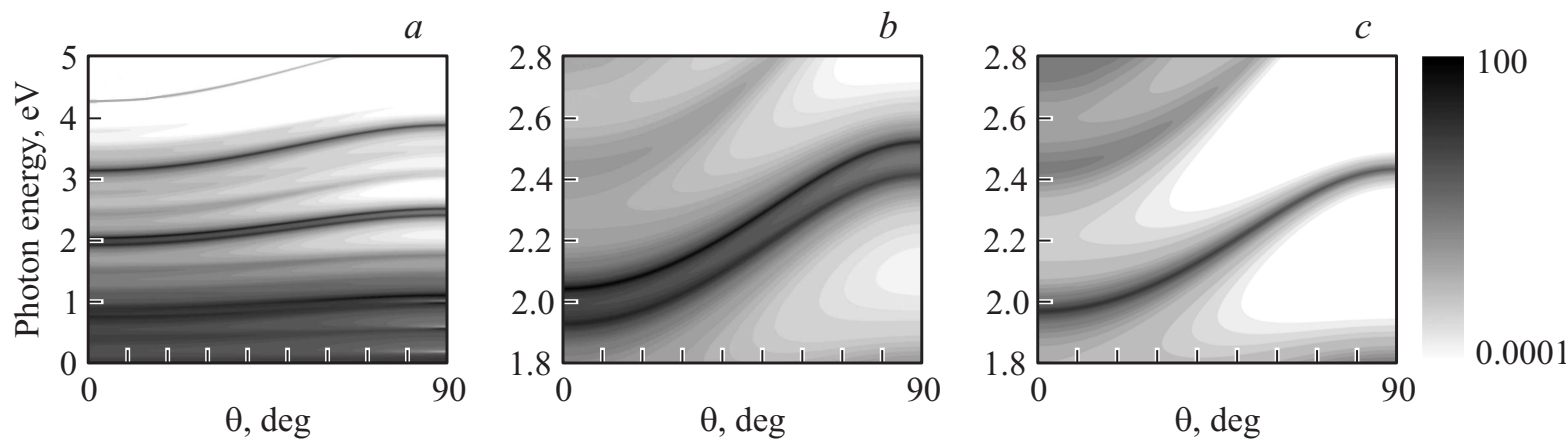

Рис. 5. Зависимость вероятности спонтанной эмиссии (нормализованной на вероятность эмиссии в свободном пространстве) для TE поляризации от частоты света и направления распространения для оптимизированного микрорезонатора с внутрирезонаторными металлическими контактами, изображенного на рис. 1. Диполь помещен в пучность электрического поля моды, изображенной на рис. $1, a, b$ или в центр структуры $c$. 
собственной моды пропорциональна величине поля в точке расположения излучателя.

Угловая зависимость пиков вероятности эмиссии соответствует брэгговскому условию для длин волн каждой из мод и совпадает с угловой зависимостью пиков в спектре отражения, приведенной на рис. 2. Дополнительные линии усиления соответствуют краевым состояниям брэгговского отражателя. Видно, в частности, что для моды, которая ранее была нами выделена как имеющая самый низкий порог генерации (рисунок $1, a$ ) наблюдается и самое высокое усиление спонтанной эмиссии на 2 порядка по сравнению со свободным пространством при нулевом нормальном излучении. Следует отметить, что эта мода имеет и самый узкий пик в спектре отражения (рис. 2) и наибольшую энергию из всех трех мод.

Таким образом, мы предложили оптимизированную структуру, которая может быть использована для создания вертикально-излучающих лазеров и других оптических устройств на основе ТП. Благодаря специфическому распределению электрического поля собственных состояний значительно уменьшено затухание внутри металлических слоев. Это достигается из-за того, что на эти слои приходятся узлы поля. Исследованы модовая структура и зависимость затухания мод от ширины слоев активной области. Найдены энергии гибридных мод, вычислены распределения электрического поля для каждой из них. Каждое распределение имеет узлы в металлических слоях, при этом две моды (первая и третья по величине энергии) - нечетные, а вторая четная. Показано, что наименьшее затухание реализуется при ширине слоев, отличной от длины волны. На длинах волн, соответствующих энергиям мод, наблюдаются пики в спектрах пропускания. При помощи методики $S$-квантования вычислена вероятность спонтанной эмиссии в предложенной структуре - для высокоэнергетической нечетной моды вероятность спонтанной эмиссии увеличивается на 2 порядка по сравнению с вероятностью в свободном пространстве.

Работа поддержана Российским научным фондом (грант № 16-12-10503) и проектом президиума РАН № 1.1.4.6, „Оптоэлектронные структуры на основе таммовских плазмонов“.

\section{Список литературы}

[1] A.P. Vinogradov, A.V. Dorofeenko, S.G. Erokhin, M. Inoue, A.A. Lisyansky, A.M. Merzlikin, A.B. Granovsky. Phys. Rev. B, 74, 045128 (2006).

[2] M. Kaliteevski, I. Iorsh, S. Brand, R.A. Abram, J.M. Chamberlain, A.V. Kavokin, I.A. Shelykh. Phys. Rev. B, 76, 165415 (2007).

[3] M.E. Sasin, R.P. Seisyan, M. Kaliteevski, S. Brand, R.A. Abram, J.M. Chamberlain, A.Yu. Egorov, A.P. Vasilev, V.S. Mikhin, A.V. Kavokin. Appl. Phys. Lett., 92 (25), 251112 (2008).
[4] T. Goto, A.V. Dorofeenko, A.M. Merzlikin, A.V. Baryshev, A.P. Vinogradov, M. Inoue, A.A. Lisyansky, A.B. Granovsky. Phys. Rev. Lett., 101, 113902 (2008).

[5] O. Gazzano, S. Michaelis de Vasconcellos, K. Gauthron, C. Symonds, J. Bloch, P. Voisin, J. Bellessa, A. Lemaitre, P. Senellart. Phys. Rev. Lett., 107, 247402 (2011).

[6] C. Symonds, G. Lheureux, J.P. Hugonin, J.J. Greffet, J. Laverdant, G. Brucoli, A. Lemaitre, P. Senellart, J. Bellessa. Nano Lett., 13 (7), 3179 (2013).

[7] O. Gazzano, S. Michaelis de Vasconcellos, K. Gauthron, S. Symonds, P. Voisin, J. Bellessa, A. Lemaitre, P. Senellart. Appl. Phys. Lett., 100, 232111 (2012).

[8] C. Symonds, A. Lemattre, P. Senellart, M.H. Jomaa, S. Aberra Guebrou, E. Homeyer, G. Brucoli, J. Bellessa. Appl. Phys. Lett., 100, 121122 (2012).

[9] K. Ding, M.T. Hill, Z.C. Liu, L.J. Yin, P.J. van Veldhoven, C.Z. Ning. Opt. Express, 21 (4) 4729 (2013).

[10] R. Brückner, A.A. Zakhidov, R. Scholz, M. Sudzius, S.I. Hintschich, H. Frob, V.G. Lyssenko, K. Leo. Nature Photonics, 6 (5), 322 (2012).

[11] M.A. Kaliteevski, A.A. Lazarenko, N.D. Il'inskaya, Yu.M. Zadiranov, M.E. Sasin, D. Zaitsev, V.A. Mazlin, P.N. Brunkov, S.I. Pavlov, A.Yu. Egorov. Plasmonics, 10 (2), 281 (2015).

[12] R. Brückner, M. Sudzius, S.I. Hintischich, H. Frob, V.G. Lyssenko, M.A. Kaliteevski, I. Iorsh, R.A. Abram, A.V. Kavokin, K. Leo. Appl. Phys. Lett., 100, 062101 (2012).

[13] D.M. Beggs, M.A. Kaliteevski, S. Brand, R.A. Abram, V.V. Nikolaev. Phys. Rev. B, 73 (3), 033106 (2006).

[14] M.A. Kaliteevski, V.A. Mazlin, K.A. Ivanov, A.R. Gubaidullin. Opt. Spectrosc., 119 (5), 832 (2015).

Редактор Г.А. Оганесян

\section{Optimization of vertical cavity laser with intra-cavity metallic layers}

\author{
A.A. Lazarenko ${ }^{1,2}$, K.A. Ivanov ${ }^{1-3}$, A.R. Gubaydullin ${ }^{1,2}$, \\ MA. Kaliteevski $1,2,4$
}

${ }^{1}$ St. Petersburg Academic University, 194021 St. Petersburg, Russia

2 ITMO University,

191707 St. Petersburg, Russia

${ }^{3}$ St. Petersburg Scientific Center,

Russian Academy of Sciences,

199034 St. Petersburg, Russia

${ }^{4}$ loffe Institute,

194021 St. Petersburg, Russia

\begin{abstract}
We have studied dependence of the decay of optical eigenmode of vertical cavity laser with intracavity metallic layers on geometric parameters of the system. It has been shown, that lowest decay corresponds to the frequency of cavity eigenmode which differ from the Bragg frequency of the Bragg reflectors forming the structure. Probability of spontaneous emission. A probability of spontaneous emission has been calculated using $S$-quantization formalism, and it has been shown, that for even high energy mode spontaneous emission rate is increased by two order of magnitude in respect to the rate in uniform space.
\end{abstract}

